\title{
Uso da terapia compressiva para prevenção e tratamento do edema
}

\author{
Use of compression therapy applied to prevention and treatment of edema \\ Uso de la terapia compresiva para la prevención y tratamiento del edema
}

Bianca Santos Soares ${ }^{1}$, Karine Costa Meloㄹ, Ianna Matos Cruz da Silva ${ }^{1}$, Wenderson Costa da Silva1, Alanna Nunes Soares ${ }^{1}$, César Augusto Pereira Souza Filho', Rafael Andrade da Silva ${ }^{1}$, Bárbara Monica Lopes e Silva1, Alexsandra Lira Sousa ${ }^{1}$, Frank Brito Frazão', Brunna Matos Sousa', Chrisllayne Oliveira da Silva ${ }^{1}$, Francisco Braz Milanez Oliveira ${ }^{1 *}$, Renan Rhonalty Rocha ${ }^{2}$, Maria Vitória Laurindo ${ }^{3}$.

\section{RESUMO}

Objetivo: analisar na literatura o uso efetivo das bandagens compressivas para controle de edemas em membros inferiores. Métodos: Trata-se de uma revisão integrativa da literatura, onde se formulou a seguinte questão clínica (PICO): "Quais evidencias cientificas recomendam o uso de técnicas compressivas para o controle do edema em membros inferiores?". Resultados: Os sete estudos incluídos nesta revisão estavam no idioma inglês (100\%). Em relação à natureza do estudo, houve prevalência de ensaios clínicos randomizados $n=4(57 \%)$. A principal linha de pesquisa investigada nessa temática versou os benefícios da terapia de compressão para tratar edema em membros inferiores. Conclusão: A terapia compressiva é considerada uma ferramenta eficaz na prevenção e controle do edema em membros inferiores, além de melhorar a qualidade de vida de pacientes edemaciados.

Palavra-chave: Edema, Extremidade Inferior, Prevenção e Controle.

\begin{abstract}
Objective: to analyze the literature on the effective use of compression bandages applied to swelling prevention and control in lower members. Methods: This is an integrative review of the literature, where the question of exercise in English (PICO) is formulated: "What are the scientific evidences recommending the use of compressive techniques for the control of edema in lower limbs?". Results: The seven studies included in this review were in the English language (100\%). Regarding the nature of the study, there was a prevalence of randomized clinical trials $n=4(57 \%)$. The main line of research investigated in this subject was the benefits of compression therapy to treat lower limb edema. Conclusion: Compression therapy is considered an effective tool in the prevention and control of lower limb edema, in addition to improving the quality of life of edematous patients.
\end{abstract}

Keywords: Edema, Lower Extremity, Prevention and Control.

\section{RESUMEN}

Objetivo: analizar en la literatura el uso efectivo de los vendajes compresivos para control de edemas en miembros inferiores. Métodos: Se trata de una revisión integrativa de la literatura, donde se formula la cuestión del ejercicio en inglés (PICO): "¿Cuáles son las evidencias científicas recomendando el uso de

${ }^{1}$ Centro Universitário de Ciências e Tecnologia do Maranhão (UniFacema). Caxias-MA. *E-mail: braz_cm@hotmail.com

2 Universidade Federal do Ceará (UFC). Sobral-CE, Brasil.

${ }^{3}$ Centro Universitário INTA (UNINTA). Sobral-CE, Brasil. 
técnicas compresivas para el control del edema en miembros inferiores?". Resultados: Los siete estudios incluidos en esta revisión se encontraban en inglés (100\%). En cuanto a la naturaleza del estudio, hubo prevalencia de ensayos clínicos aleatorizados (4/57\%). La principal línea de investigación investigada en esta temática versó los beneficios de la terapia de compresión para tratar edema en miembros inferiores. Conclusión: La técnica compresiva es considerada una herramienta eficaz en la prevención y control del edema en miembros inferiores, además de mejorar la calidad de vida de pacientes edemaciados.

Palabra clave: Edema, Extremidad Inferior, Prevención e Control.

\section{INTRODUÇÃO}

Edema é definido como o aumento no volume do fluido no interstício que se manifesta clinicamente por uma fóvea ao pressionar contra uma proeminência óssea. Na síndrome nefrótica, insuficiência hepática, desnutrição, síndrome de má absorção e perda de proteína, enteropatia, o edema é generalizado, bilateral, macio e simétrico o denominador comum é a hipoalbuminemia, que condiciona a diminuição de Pressão oncótica e produção de fluidos intravasculares ao interstício, o que diminui o volume circulação sanguínea circulante, estimulando a sistema renina-angiotensina-aldosterona, bem quanto à arginina vasopressina, causando retenção de água e sódio (VILLEGAS BF, et al., 2014).

Em uma pessoa de tamanho médio o excesso de líquidos aproximados necessários para ser classificado como um edema aparente é de 2,3 a $4,5 \mathrm{~kg}$ ou quando o fluido intersticial representar mais de $10 \%$ do peso corporal do indivíduo (VILLEGAS BF, et al., 2014).

O edema periférico de membros inferiores está associado ainda a permanência em uma postura prolongada, seja ela sentada ou em pé, principalmente nas atividades de trabalho, esse fator, deve ser considerado agravante para complicações circulatórias (BRITO APNP, et al., 2013).

De acordo com Parsch H (2017), a compressão não só reduz a filtração do fluido, mas também previne a adesão dos leucócitos às células endoteliais como um primeiro passo da inflamação. Se um efeito mecânico nas veias for alcançado, pressões muito maiores são necessárias, especialmente na posição vertical. As principais características da compressão que melhoram a hemodinâmica venosa são uma pressão tolerável em repouso e uma alta pressão na posição vertical para contrabalançar e favorecer a circulação de repouso. Em contraste com os têxteis elásticos, as ataduras inelásticas podem permanecer na perna por até uma semana ou até mais, e a maioria dos componentes principais pode ser lavada e reutilizada.

Os benefícios das bandagens compressivas incluem melhor qualidade de vida para o paciente e economias associadas em tempo e recursos de enfermagem, onde ressalta-se que a escolha do melhor método compressivo varia de acordo com a avaliação do paciente, que deve ocorrer de forma holística e atender as suas necessidades individuais. Atualmente o método de bandagem com dispositivos de compressão de velcro ajustáveis, tem se destacado por maior redução de linfedemas, permitindo que os pacientes diminuam o numero de consultas medicas e por serem de fácil uso (HUNTER M, 2017).

Existindo vários sistemas de bandagens, meias e wrap-around disponíveis, que visam reduzir edema, apoiando o movimento de fluidos dos tecidos de volta para as veias profundas através de estímulos físicos (MOSTI G, 2017).

Portanto, este estudo teve como objetivo analisar na literatura o uso efetivo das bandagens compressivas para controle de edemas em membros inferiores.

\section{METODOLOGIA}

Trata-se de uma revisão integrativa da literatura. Sua elaboração inclui: definição do objetivo; estabelecimento de critérios de inclusão e exclusão para a seleção da amostra; definição das informações a serem extraídas dos artigos selecionados; análise, e discussão dos resultados. Para orientar este estudo, 
formulou-se a seguinte questão clínica (PICO): Quais evidencias cientificas recomendam o uso de técnicas compressivas para o controle do edema em membros inferiores?

A estratégia PICO, que representa a População ou Problema (P), Intervenção (I), Comparador (C) e o Desfecho $(\mathrm{O})$, foi utilizada para a construção da questão dirigente desta revisão integrativa da literatura. Para que fossem encontrados os estudos relevantes, que respondessem à pergunta de pesquisa, utilizou-se de descritores indexados nos idiomas português, inglês e espanhol. Os descritores foram obtidos a partir do Medical Subject Headings (MESH), dos Descritores em Ciências da Saúde (DeCS) e dos Títulos Cumulative Index to Nursing and Allied Health Literature (CINAHL), onde neste último, tivemos que remover o elemento $\mathrm{O}$, para encontrar um número maior de estudos.

A coleta de dados foi executada por meio de busca eletrônica no período de abril de 2018 nas seguintes bases de dados: Biblioteca Virtual de Saúde - BVS (Bireme), National Library of Medicine (PubMed) e Cumulative Index to Nursing and Allied Health Literature (CINAHL). Os critérios de inclusão definidos foram: estudos primários, disponíveis em sua totalidade, publicados nos últimos 10 anos, de 2008 até 2018, nos idiomas Português, Espanhol e Inglês, textos completos, além de estudos realizados com humanos. Foram excluídos da busca inicial capítulos de livros, resumos, textos incompletos, teses de doutorado, dissertações de mestrados, monografias e relatos técnicos. Utilizaram-se os seguintes bancos com seus respectivos descritores (Quadro 1).

Quadro 1 - Elementos da estratégia pico e descritores utilizados, Caxias, MA, 2018.

\begin{tabular}{|c|c|c|c|c|}
\cline { 2 - 5 } \multicolumn{1}{c|}{} & Elementos & Mesh & Decs & Títulos Cinahl \\
\hline $\mathbf{P}$ & "Edema" & "Edema" & "Edema" & "Edema" \\
\hline $\mathbf{I}$ & $\begin{array}{c}\text { "Bandagens } \\
\text { compressivas" }\end{array}$ & $\begin{array}{c}\text { "Compression } \\
\text { Bandages" }\end{array}$ & $\begin{array}{c}\text { "Bandagens } \\
\text { compressivas" }\end{array}$ & $\begin{array}{c}\text { "Elastic Bandages" } \\
\text { "Compression Therapy" }\end{array}$ \\
\hline $\mathbf{C}$ & $\mathrm{X}$ & $X$ & $X$ & $X$ \\
\hline $\mathrm{O}$ & "Controle" & $\begin{array}{c}\text { "Prevention and } \\
\text { control" }\end{array}$ & $\begin{array}{c}\text { "Prevenção \& } \\
\text { Controle" }\end{array}$ & $X$ \\
\hline
\end{tabular}

Fonte: Autoria Própria, 2018.

Os termos utilizados durante a pesquisa foram classificados e combinados nos bancos de dados, resultando em estratégias específicas de cada base (Quadro 2).

Quadro 2 - Estratégias de busca utilizadas nas bases de dados BIREME, PubMed e CINAHL. Caxias, MA, 2018.

\begin{tabular}{|c|l|c|c|c|}
\hline Base de dados & \multicolumn{1}{|c|}{ Estratégia de busca } & R & F & S \\
\hline $\begin{array}{c}\text { Bireme } \\
\text { (descritores } \\
\text { Decs) }\end{array}$ & $\begin{array}{l}\text { (tw:(Edema)) AND (tw:(Bandagens compressivas)) AND } \\
\text { (tw:(Prevenção \& and controle)) }\end{array}$ & 17 & 12 & 1 \\
\hline $\begin{array}{c}\text { PubMed } \\
\text { (descriptors }\end{array}$ & $\begin{array}{l}\text { (("edema"[All Fields] OR "edema"[MeSH Terms] OR "edema"[All } \\
\text { Fields]) AND ("compression bandages"[MeSH Terms] OR } \\
\text { ("compression"[All Fields] AND "bandages"[All Fields]) OR }\end{array}$ & & & \\
& $\begin{array}{l}\text { "compression bandages"[All Fields])) AND ("prevention and } \\
\text { control"[Subheading] OR ("prevention"[All Fields] AND "control"[All }\end{array}$ & & \\
& $\begin{array}{l}\text { Fields]) OR "prevention and control"[All Fields]) AND ("loattrfree } \\
\text { full text"[sb] AND "2008/05/25"[PDat] :"2018/05/22"[PDat]) }\end{array}$ & & \\
\hline $\begin{array}{l}\text { CINAHL } \\
\text { (CINAHL } \\
\text { Headings) }\end{array}$ & $\begin{array}{l}\text { ((MM "Edema+")) AND (MM "Compression Therapy" OR MM } \\
\text { "Elastic Bandages" AND MM "Edema+) }\end{array}$ & & \\
\hline
\end{tabular}

Fonte: Base de dados, 2018.

$\mathrm{R}=$ resultado; $\mathrm{F}=$ Filtrados; $\mathrm{S}=$ Selecionados. 
Figura 1 - Fluxo do processo de seleção dos estudos para a revisão integrativa. Caxias, MA, 2018. ( $n=7)$.

Questăo norteadora: Ouais evidencias cientificas comprovam o uso de técnicas compressivas para o controle do edema em membros inferiores?

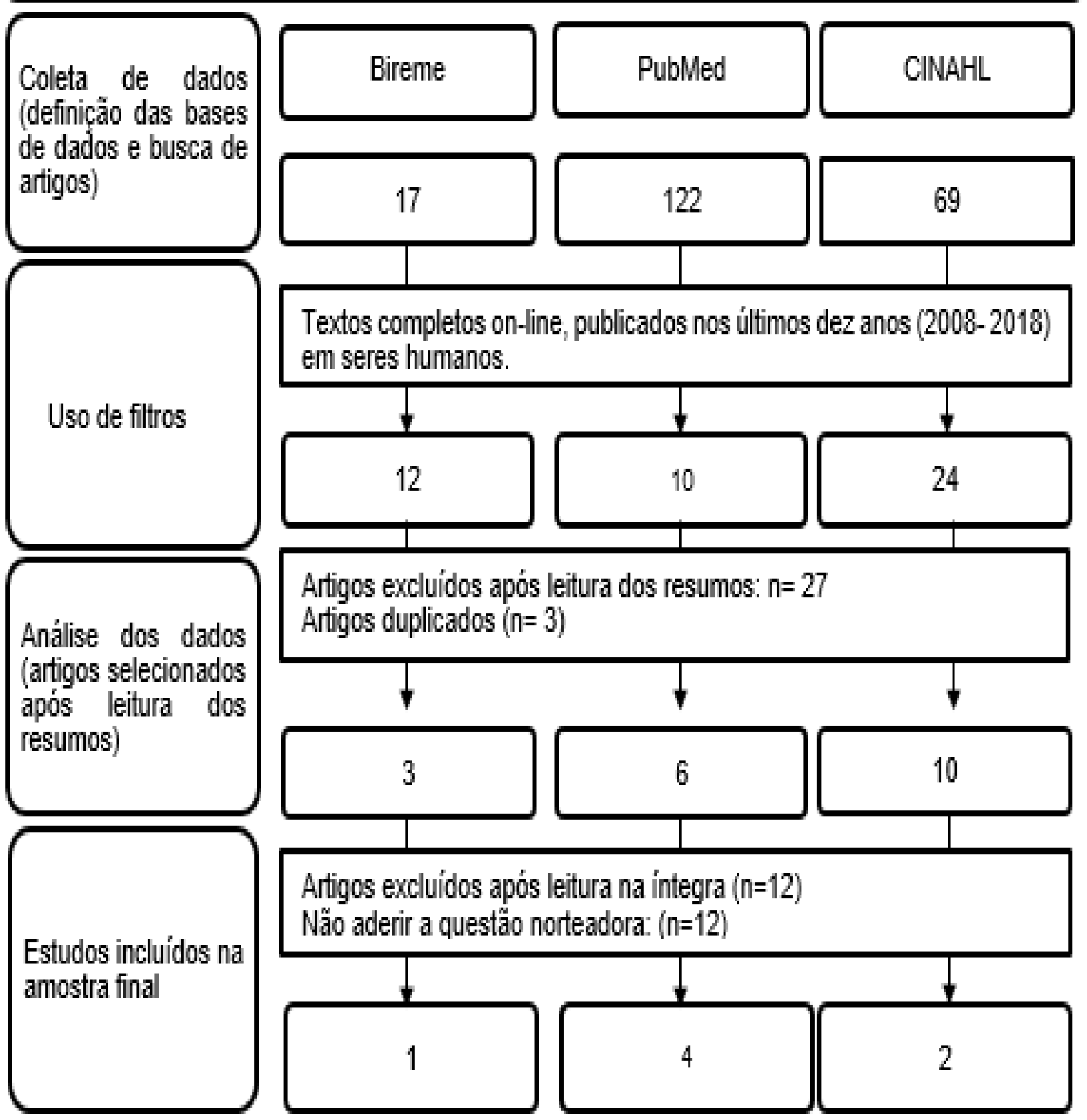

Fonte: Base de dados, 2018. $\mathrm{N}$ = número de artigos selecionados.

\section{RESULTADOS}

Os sete estudos incluídos nesta revisão estavam no idioma inglês (100\%). Em relação à natureza do estudo, houve prevalência de ensaios clínicos randomizados $n=4(57 \%)$. A principal linha de pesquisa investigada nessa temática versou os benefícios da terapia de compressão para tratar edema em membros inferiores (Quadro 3). Os estudos avaliaram a aplicação de terapia de compressão para prevenir ou minimizar edema em membros inferiores (Quadro 4). 
Quadro 3 - Distribuição das publicações incluídas segundo o título, ano de publicação, país onde o estudo foi realizado, delineamento da pesquisa, nível de evidência e grau de recomendação, Caxias, MA, 2018.

\begin{tabular}{|c|c|c|c|c|c|c|c|}
\hline $\begin{array}{l}\text { № de } \\
\text { ordem }\end{array}$ & $\begin{array}{l}\text { Autores/ano } \\
\text { de publicação }\end{array}$ & Título & Base de dado & Pais & $\begin{array}{l}\text { Delineamento da } \\
\text { pesquisa }\end{array}$ & $\begin{array}{l}\text { Nível de } \\
\text { evidência }\end{array}$ & $\begin{array}{l}\text { Grau de } \\
\text { recomendação }\end{array}$ \\
\hline A.1 & $\begin{array}{l}\text { AUCKEL JPH, } \\
\text { et al., } 2009 .\end{array}$ & $\begin{array}{l}\text { To Wear or not Wear Compression } \\
\text { Stockings after Varicose Vein Stripping: } \\
\text { A Randomised Controlled Trial. }\end{array}$ & PUBMED & Holanda & $\begin{array}{l}\text { Ensaio clínico } \\
\text { randomizado }\end{array}$ & 2 & $\mathrm{C}$ \\
\hline A.2 & XU B, 2010 & $\begin{array}{l}\text { DVT in acute stroke the use of } \\
\text { graduated compression stocking }\end{array}$ & PUBMED & Austrália & Revisão da liteatura & 5 & $\mathrm{C}$ \\
\hline A.3 & $\begin{array}{l}\text { MOSTI G E } \\
\text { PARTSCH H, } \\
2013\end{array}$ & $\begin{array}{l}\text { Occupational Leg odema is More } \\
\text { Reduced by Antigraduated than by } \\
\text { Graduated Stockings }\end{array}$ & PUBMED & Amsterdã & Estudo de Coorte & 3 & A \\
\hline A.4 & $\begin{array}{l}\text { SUGISAWA R, } \\
\text { et al., } \\
2016\end{array}$ & $\begin{array}{l}\text { Effects of Compression Stockings on } \\
\text { Elevation of Leg Lymph Pumping } \\
\text { Pressure and Improvement of Quality of } \\
\text { Life in Healthy Female Volunteers: A } \\
\text { Randomized Controlled Trial }\end{array}$ & PUBMED & Japão & $\begin{array}{l}\text { Ensaio clínico } \\
\text { randomizado }\end{array}$ & 2 & A \\
\hline A.5 & $\begin{array}{l}\text { MARIANI F, et } \\
\text { al., } 2011\end{array}$ & $\begin{array}{l}\text { Multicenter randomized trial comparing } \\
\text { compression with elastic stocking } \\
\text { versus bandage after surgery for } \\
\text { varicose veins }\end{array}$ & BIREME & Itália & $\begin{array}{l}\text { Ensaio clínico } \\
\text { randomizado }\end{array}$ & 2 & $A$ \\
\hline A. 6 & $\begin{array}{l}\text { MATHEWS S, } \\
\text { et al., } 2015\end{array}$ & $\begin{array}{l}\text { Effect of elastic bandage wraps on leg } \\
\text { edema in patients before and after liver } \\
\text { transplant }\end{array}$ & CINAHL & Geórgia & $\begin{array}{l}\text { Ensaio clínico } \\
\text { randomizado }\end{array}$ & 2 & $A$ \\
\hline A.7 & $\begin{array}{l}\text { ATKIN L, et al., } \\
2018\end{array}$ & $\begin{array}{l}\text { Diabetic foot ulceration: the impact of } \\
\text { oedema }\end{array}$ & CINAHL & $\begin{array}{l}\text { Reino } \\
\text { Unido }\end{array}$ & Estudo de Coorte & 3 & $A$ \\
\hline
\end{tabular}

A = artigo. Nível de evidência: 1- Revisão sistemática; 2-Ensaio clinico randomizado; 3- Estudos de Coorte; 4- Estudos de caso e controle; 5- Estudos de casos; 6 - Estudos Transversais; 7- Opinião de especialista. Grau de recomendação: A - recomenda intervenção dos resultados; B - os resultados não conclusivos e ineficientes para recomendar intervenção; C - resultado contraindica intervenção.

Fonte: Autoria própria, 2018. 
Quadro 4 - Publicações incluídas segundo objetivo principal, perfil amostral e principais resultados, Caxias, MA, 2018.

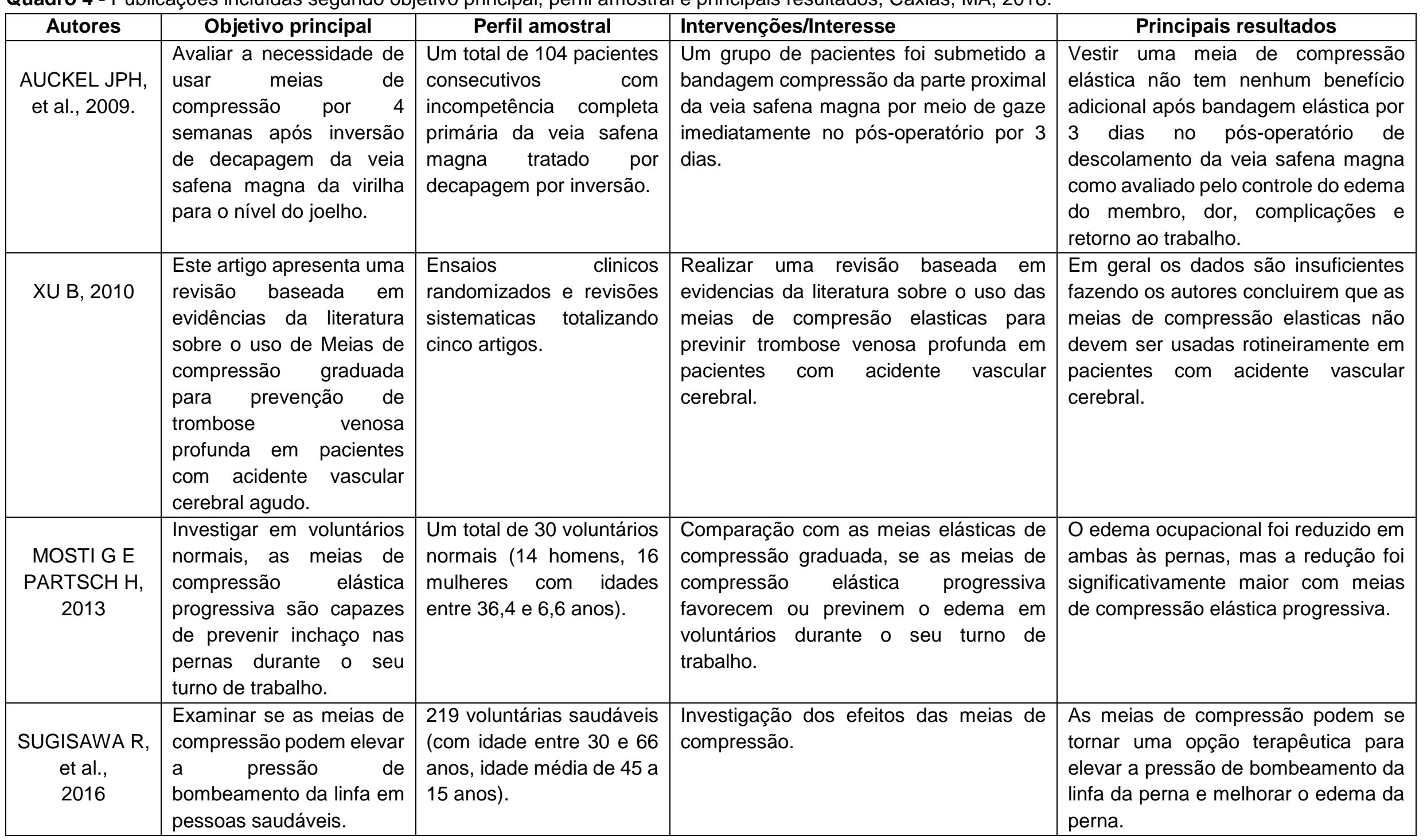

REAS/EJCH | Vol. Sup.27 | e754 | DOI: https://doi.org/10.25248/reas.e754.2019 Página 6 de 10 


\begin{tabular}{|c|c|c|c|c|}
\hline $\begin{array}{l}\text { MARIANI F, et } \\
\quad \text { al., } 2011\end{array}$ & $\begin{array}{l}\text { Avaliar a eficácia de um } \\
\text { novo kit de meia usado } \\
\text { para compressão de } \\
\text { membro no pós- } \\
\text { operatório. }\end{array}$ & $\begin{array}{l}60 \text { pacientes submetos à } \\
\text { cirurgia unilateral de } \\
\text { varizes. }\end{array}$ & $\begin{array}{l}\text { Por duas semanas no pós-operatório, } 30 \\
\text { pacientes usaram a meia de Sigvaris dia } \\
\text { e noite, e } 30 \text { pacientes foram tratados } \\
\text { com a terapia de compressão de curativo } \\
\text { de curta duração. }\end{array}$ & $\begin{array}{l}\text { Os pacientes tratados com meias têm } \\
\text { menos edema em comparação com } \\
\text { aqueles tratados com bandagem } \\
\text { padrão, e a aplicação do kit de } \\
\text { estocagem melhora a adesão do } \\
\text { paciente à terapia compressiva pós- } \\
\text { operatória. }\end{array}$ \\
\hline $\begin{array}{l}\text { MATHEWS S, } \\
\text { et al., } 2015\end{array}$ & $\begin{array}{l}\text { Para determinar se o uso } \\
\text { de envoltórios de } \\
\text { bandagem elástica da } \\
\text { perna em pacientes antes } \\
\text { e após o transplante de } \\
\text { fígado diminui a formação } \\
\text { de edema e dor na perna. }\end{array}$ & $\begin{array}{l}24 \text { pacientes antes e } \\
\text { depois de um transplante } \\
\text { de fígado. }\end{array}$ & $\begin{array}{l}\text { Envoltório de bandagem elástica aplicado } \\
\text { na perna por } 8 \text { horas. }\end{array}$ & $\begin{array}{l}\text { A aplicação de bandagem elástica } \\
\text { envolve uma perna por um período de } \\
8 \text { horas, levou a uma redução } \\
\text { significativa da formação de edema e } \\
\text { da dor em comparação com a perna } \\
\text { não envolvida. }\end{array}$ \\
\hline $\begin{array}{l}\text { ATKIN L, et } \\
\text { al., } 2018\end{array}$ & $\begin{array}{l}\text { Oferecer uma visão geral } \\
\text { de abordagens de } \\
\text { terapias de compressão } \\
\text { em pacientes que } \\
\text { apresentam úlcera do pé } \\
\text { diabético em combinação } \\
\text { com edema de membros } \\
\text { inferiores. }\end{array}$ & $\begin{array}{l}\text { Ocorreu por um período } \\
\text { de } 12 \text { semanas. Dados de } \\
7 \\
\text { Clínicas de podologia } \\
\text { foram coletadas e um total } \\
\text { de } 80 \text { pacientes, } \\
\text { selecionados } \\
\text { atoriamente. }\end{array}$ & $\begin{array}{l}\text { Discutir os benefícios de kits de } \\
\text { compressão de meias, envoltórios de } \\
\text { compressão, } \\
\text { multicompondagem } \\
\text { hipoglicemia e com baixo teor de } \\
\text { gorduras, com o paciente. }\end{array}$ & $\begin{array}{l}\text { O sistema envoltório de compressão } \\
\text { pode conter vantagens em pacientes } \\
\text { onde o volume de membros irá reduzir } \\
\text { rapidamente. }\end{array}$ \\
\hline
\end{tabular}

Fonte: Autoria própria, 2018. 


\section{Discussão e tipos de técnicas compressivas}

Dentre os diversos tipos de técnicas compressivas, é primordial escolher a mais adequada para a necessidade do paciente, os artigos abordam justamente sobre o assunto e trazem comparações acerca da melhor escolha. Foi realizado um estudo comparando meias de compressão elástica progressiva e meias elásticas de progressão graduada em tratamento de edema ocupacional (relacionado ao tempo de turno de trabalho) e foi comprovado que as meias de compressão elástica progressiva são mais confortáveis, mais fáceis de colocar e mais eficazes na melhoria da função de bombeamento venoso. Apesar dessas vantagens, um gradiente de pressão inversa poderia teoricamente produzir um obstáculo ao retorno venoso ao nível da panturrilha em indivíduos não ativos, ter um efeito negativo na prevenção e no tratamento do edema ou até favorecer a sua formação (MOSTI G e PARTSCH H, 2013).

Quanto aos pacientes que passaram por algum tipo cirurgia para remoção de veias varicosas, foi realizada uma comparação entre os que utilizaram meias compressão elástica e ataduras no pós-operatório (MARIANI F, et al., 2011; AUCKEL JPH, et al., 2009), onde se verificou que os pacientes toleraram mais o uso das meias.

Outro caso analisado foi o uso da de meias compressivas para ajudar na cicatrização de ulceração em membros inferiores, onde a mesma se mostrou eficaz e ficou claro que os pacientes devem ser orientados quanto ao seu uso de forma correta (ATKIN L, et al., 2018).

Evidências mostraram que na população em geral, há um número considerável de pessoas que se queixam de edema nas pernas decorrente de algumas doenças do envelhecimento populacional como, diabetes hipertensão, problemas nos rins, etc., onde $10 \%$ das pessoas que são acometidas pelo problema adquirem ulcerações nas pernas e possuem um difícil tratamento em decorrência de linfedemas, do aumento das taxas de obesidade ou até mesmo depois de trabalhar por períodos prolongados na posição sentada ou em pé. Com base nisso, as meias de compressão estão sendo indicadas para melhorar o edema nas pernas, pois sua aplicação com uma pressão e comprimento adequados, asseguram que menos fluidos linfáticos vazem para fora dos vasos capilares, estimulando simultaneamente que mais fluidos sejam reabsorvidos pelos sistemas venosos e linfáticos reduzindo assim o edema (ATKIN L, et al., 2018; XU B, 2010; SUGISAWA $\mathrm{R}$, et al., 2016).

As meias de compressão apresentam vários outros benefícios, que incluem aos pacientes não restringirem as escolhas dos calçados ou do vestuário; oferece níveis garantidos de compressão, permite aos pacientes o autocuidado, previne o aparecimento de trombose venosa profunda, reduz a dor corporal, reduz a ocorrência de câimbras, ajuda na cicatrização de úlceras em pacientes com ulceração na perna, ajuda no tratamento de veia varicosa, reduz edema pós-cirurgias e diminui o tempo de atuação da enfermagem para vestir, pois as meias são mais rápidas para serem aplicadas, consequentemente levando a uma melhor qualidade na saúde dos pacientes (MATHEWS S, et al., 2015; XU B, 2010; MARIANI F, et al., 2011; AUCKEL JPH, et al., 2009).

\section{Implicações clínica e manejo do edema}

O edema em membros inferiores é encontrado rotineiramente por muitos profissionais. O peso extra do membro edemaciado pode afetar a deambulação e a mobilidade, provocando dores nas articulações e ao redor dos músculos. As técnicas de compressão do edema podem ser a melhor maneira de gerenciar a sua evolução (MATHEWS S, et al., 2015; ATKIN L, et al., 2018).

A simples aplicação de compressão no edema de membros inferiores, pode reduzir tanto o edema quanto as dores nos membros, desta forma melhorando a mobilidade do indivíduo, e consequentemente levando a uma melhor qualidade de vida (MOSTI G e PARTSCH H, 2013; MATHEWS S, et al., 2015; ATKIN L, et al., 2018; SUGISAWA R, et al., 2016).

De acordo com Atkin L (2018), no diabetes esse peso extra do membro afetará a cicatrização da ulceração do pé diabético. A terapia compressiva é a base do gerenciamento do edema e, com o envolvimento correto da equipe especializada, monitoramento regular e um plano de tratamento adaptado a cada paciente, esta prática é segura em pacientes diabéticos com circulação arterial periférica adequada. 
As pernas podem inchar também fisiologicamente em voluntários normais que trabalham sentados ou em pé. Como citam Mosti G e Partsch H (2013) e Sugisawa R, et al. (2016), considera-se que o edema ocupacional da perna se desenvolve como resultado da gravidade aumentando a pressão hidrostática e aumentando a pressão venosa nas pernas, o que, por sua vez, leva ao extravasamento de líquido das vênulas, causando o edema. As meias de compressão elástica progressiva causaram uma redução significativamente do edema ocupacional medido pela volumetria da perna em indivíduos saudáveis.

Na pesquisa de Mathews S, et al. (2015) evidenciaram que a aplicação de bandagem elástica levou a uma redução significativa da formação de edema e da dor em comparação com a perna não envolvida em pacientes submetidos ao transplante de fígado. Já na pesquisa de Mariani F, et al. (2011) realizada com pacientes submetidos a cirurgia de varizes, evidenciaram que os pacientes tratados com meias têm menos edema em comparação com aqueles de bandagem padrão. No entanto, os pacientes com doença vascular periférica grave não podem usar meias. Nesse caso, uma atadura rígida ou curta seria indicada, se tolerada pelo paciente.

\section{Tempo de uso das técnicas compressivas}

O tempo de uso indicado para as bandagens compressivas está relacionado com as características de cada caso, desta forma o tempo de uso deverá ser adaptado de acordo com suas especificidades de cada paciente (MARIANI F, et al., 2011). No estudo de Mathews S, et al. (2015) a aplicação de bandagem elástica por um período de 8 horas, em pacientes que foram submetidos a transplante de fígado, levou a uma redução significativa da formação de edema e da dor em comparação com a perna não envolvida. Já no estudo de Mariani F, et al. (2014) submetidos a cirurgia de varizes, nenhum edema foi encontrado em $80 \%$ dos pacientes que usaram kit de meia durante 14 dias.

Na pesquisa de Auckel JPH (2009), quando utilizadas as meias elásticas no período do pré-operatório por seis meses e com uma compressão reduzida o método se torna mais confortável e eficaz no controle de hematomas e tromboflebites. Em pacientes mulheres saudáveis com redução da pressão de bombeamento da linfa, Sugisawa R, et al. (2016) evidenciaram que o uso das meias compressivas nas pernas bilaterais diariamente, da manhã até a hora de dormir, durante o período de estudo de 16 semanas melhorou a pressão de bombeamento da linfa e o edema nas pernas.

Elencou-se um conjunto de quatro intervenções de enfermagem necessárias para prevenção e controle de edema em membros inferiores organizados com seus respectivos níveis de evidencia, podem ser visualizados, no Quadro 5.

Quadro 5 - Bundles de Intervenções para prevenção e controle de edema em membros inferiores. CaxiasMA, 2018.

\begin{tabular}{|l|c|}
\hline INTERVENÇÕES & Nível de evidência \\
\hline Aplicação de técnicas compressivas no pós-operatório & II \\
\hline Usar terapia de compressão para evitar edema ocupacional & III \\
\hline Aplicar emolientes nos membros inferiores para hidratação & III \\
\hline Elevar os membros inferiores & III \\
\hline
\end{tabular}

Fonte: Autoria própria, 2018.

\section{DISCUSSÃO}

\section{Bundles de Intervenções}

Essas cinco intervenções, apresentam fortes evidencias com relação a sua efetividade, para prevenção e controle do edema em membros inferiores. Entretanto, para potencializar efeitos de cada uma dessas intervenções de enfermagem serão expostas cada uma elencando sua importância. 


\section{Aplicação de técnicas compressivas no pós-operatório}

A compressão dos membros inferiores no pós-operatório é amplamente utilizada para evitar ou reduzir edema e dor, a maioria dos cirurgiões prescrevem alguma técnica de compressão com uma duração variável após o procedimento cirúrgico, sem diferença significativa entre uma, duas ou seis semanas de compressão foi encontrado para o controle das complicações pós-operatórias, sendo aconselhável sua utilização mínima de 1 semana (MARIANI F, et al., 2011).

\section{Usar terapia de compressão para evitar edema ocupacional}

As meias de compressão elástica exercem uma pressão progressiva, maior na panturrilha do que no tornozelo, sendo uma técnica fácil de executar, confortável e mais eficaz na melhoria da função de bombeamento venoso tendo como resultado uma diminuição significativa do edema ocupacional em comparação a outras técnicas (MOSTI G e PARTSCH H, 2013).

\section{Aplicar emolientes nas pernas, a fim de manter uma boa hidratação}

Cuidados com a pele é uma parte essencial para o manejo de pacientes com edema de membros inferiores, pois a pele saudável, hidratada e intacta fornece uma barreira mais eficaz para reduzir o risco de infecção num paciente já imunocomprometidos. Assim os cuidados com a pele devem ser incorporados na prática diária dos pacientes, onde os mesmos devem ser incentivados a construir uma rotina diária incluindo inspeção da pele, lavagem cuidadosa e uso de emolientes (ATKIN L, et al., 2018).

\section{Elevar membros inferiores}

Os pacientes devem ser encorajados a elevar suas pernas por períodos curtos durante o dia e quando forem dormir na cama à noite. Pois os períodos de elevação dos membros inferiores ajudam retorno venoso e linfático, diminuindo o edema evitando assim outras complicações como trombose (ATKIN L, et al., 2018).

\section{CONSIDERAÇÕES FINAIS}

Este estudo permitiu compreender que a técnica compressiva é considerada uma ferramenta eficaz na prevenção e controle do edema em membros inferiores, além de melhorar a qualidade de vida de pacientes edemaciados. Torna-se claro, a necessidade de escolher a melhor técnica compressiva disponível. Pretendese com esse estudo gerar informações para os avanços na técnica compressiva quanto a prevenção e controle do edema em membros inferiores, abordando o problema e buscando precocemente intervenções para minimizar complicações da má conduta terapêutica dessa pratica.

\section{REFERÊNCIAS}

1. ATKIN L, et al. Diabetic foot ulceration: the impacto of oedema. Wounds UK, 2018; 14(1): 33-39.

2. BRITO APNP, et al. Investigação de edema postural de membros inferiores em agentes de trânsito. J Vasc Bras, 2013; 12(4): 289295.

3. AUCKEL JPH, et al. To wear compression stockings after varicose vein stripping: A randomised controlled trial. Eur J Vasc Endovasc Srg, 2009; 38(3): 387-391.

4. HUNTER M. Adjustable velcro compression devices and management of lower limb lymphoedema. JCN, 2017; 31(5): 20-28.

5. MARIANI F, et al. Multicenter randomized trial comparing compression with elastic stocking versus bandage after surgery for varicose veins. J Vascu Surg, 2011; 53(1): 155-22.

6. MATHEWS S, et al. Effect of elastic bandage wraps on leg edema in patients before and after liver transplant. Progr in Transplan, 2015; 25(4): 302-306.

7. MOSTI G. Compression therapy in the community. JCN, 2017; 31(5): 36-42.

8. MOSTI G, PARTSCH H. Occupational leg Oedema is more reduced by ant gradated than by graduated stockings. J Vasc Vascu Surg, 2013; 45: 523-527.

9. PARTSCH H. Inelastic compression by bandages: effective but requiring education. Wounds international, 2017; 8(3): 6-9.

10. PARTSCH H, MOTIMER P. Compression for leg wounds. J Demartol, 2015; 173(2): 359-369.

11. SUGISAWA R, et al. Effects of compression stockings on elevation of leg lymph pumping pressure and improvement of quality of life in healthy female volunteers: A randomized controlled trial. Lymphatic Resear Biolo, 2016; 14(2): 95-103.

12. VILLEGAS BF, et al. Edema: Enfoque clinico. Med Int Méx, 2014; 30(1): 51-55.

13. XU B. DVT in acute stroke the se of gradated compression stockings. Reprinted from Astralian Family Physician, 2010; 39(7): 485487. 